\title{
Design of Multimodal Absorption in the Mid-IR: A Metal Dielectric Metal Approach
}

\author{
Nelson W. Pech-May,* Tobias Lauster, and Markus Retsch* \\ Cite This: ACS Appl. Mater. Interfaces 2021, 13, 1921-1929 \\ Read Online
}

ABSTRACT: Specific control on the mid-infrared (mid-IR) emission properties is attracting increasing attention for thermal camouflage and passive cooling applications. Metal-dielectric-metal (MDM) structures are well known to support strong magnetic polariton resonances in the optical and near-infrared range. We extend the current understanding of such an MDM structure by specifically designing Au disc arrays on top of $\mathrm{ZnS}-\mathrm{Au}-\mathrm{Si}$ substrates and pushing their resonances to the mid-IR regime. Therefore, we combine fabrication via lift-off photolithography with the finite element method and an inductance-capacitance model. With this combination of techniques, we demonstrate that the magnetic polariton resonance of the first order strongly depends on the individual disc diameter. Furthermore, the fabrication of multiple discs within one unit cell allows a linear combination of the fundamental resonances to

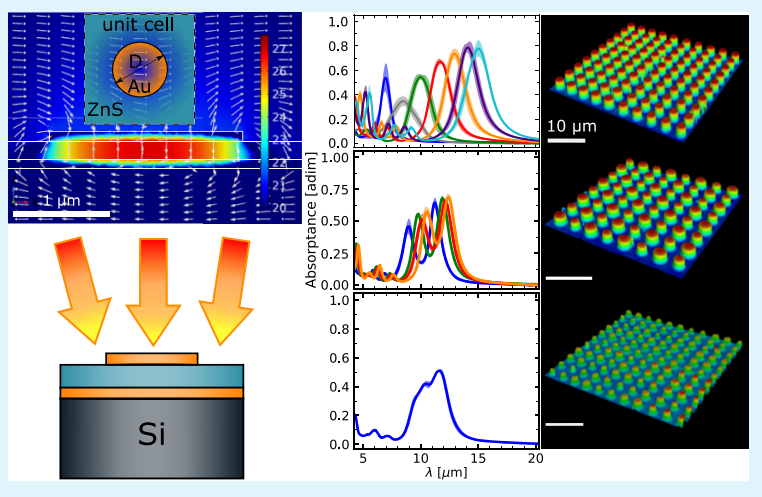
conceive broadband absorptance. Quite importantly, even in mixed resonator cases, the absorptance spectra can be fully described by a superposition of the individual disc properties. Our contribution provides rational guidance to deterministically design mid-IR emitting materials with specific narrow- or broadband properties.

KEYWORDS: metal-dielectric-metal periodic structures, mid-IR absorption, magnetic polariton absorption, multimodal absorption, broadband absorption, thermal emission, radiative cooling, microfabrication

\section{INTRODUCTION}

Infrared (IR) absorber metamaterials find applications in thermophotovoltaics, near-infrared (NIR) cloaking, thermal camouflage, and several fields of basic and applied research. ${ }^{1-9}$ Accordingly, several metamaterial design concepts have been explored to produce single-band, multiple-band, and broadband absorptance in the IR region of the electromagnetic (EM) spectrum. ${ }^{1,2,6}$ However, most studies have focused on the NIR, and very few attempts can be found in the midinfrared (mid-IR) or far-infrared. ${ }^{1,2}$ The main challenges are the available fabrication techniques and the understanding of the physical nature of the metamaterial interaction with incoming EM radiation. The former is often time consuming or hardly scalable, and the latter can provide the right parameters (geometrical and optical properties) required to address the spectral region of interest. Such engineered IR absorbers are usually the principal components in cutting-edge technologies such as artificial IR camouflage. In this case, metamaterials based on metal-dielectric-metal (MDM) structures have been used to obtain a strong absorptance peak by excitation of magnetic polariton resonances. Accordingly, demonstrations of IR camouflage have been realized using an MDM structure with a high absorptance peak in the 3-5 $\mu \mathrm{m}$ spectral region and low absorptance in the $8-$ $14 \mu \mathrm{m}$ detectable range of the IR camera used. ${ }^{1,8}$ Similarly, surface-enhanced IR spectroscopy for sensing from NIR to mid-IR has been successfully demonstrated using self-similar arrays of multiresonant plasmonic nanoantennas. ${ }^{2}$ Moreover, experimental observation of localized surface phonon polariton resonances has been reported in the mid-IR using nanofabricated, periodic arrays of cylindrical $6 \mathrm{H}-\mathrm{SiC}$ nanopillars. This promises a potential contribution to the development of components for mid-IR free-space communications. ${ }^{6}$

Likewise, the engineering of absorption and emission properties in the mid-IR range is of great importance for the field of passive cooling devices. The relevant wavelength range is located around the maximum of the spectral radiance of a black body radiator at about $300 \mathrm{~K}$. This can be considered as a reference temperature, at which most terrestrial structures emit thermal radiation at around $10 \mu \mathrm{m}$-depending on their emissivity. Passive radiative cooling utilizes thermal radiation as a way to transfer terrestrial heat into the outer space, which serves as a heat sink. Particularly, the spectral window from 7.5

Received: October 13, 2020

Accepted: December 22, 2020

Published: January 4, 2021 

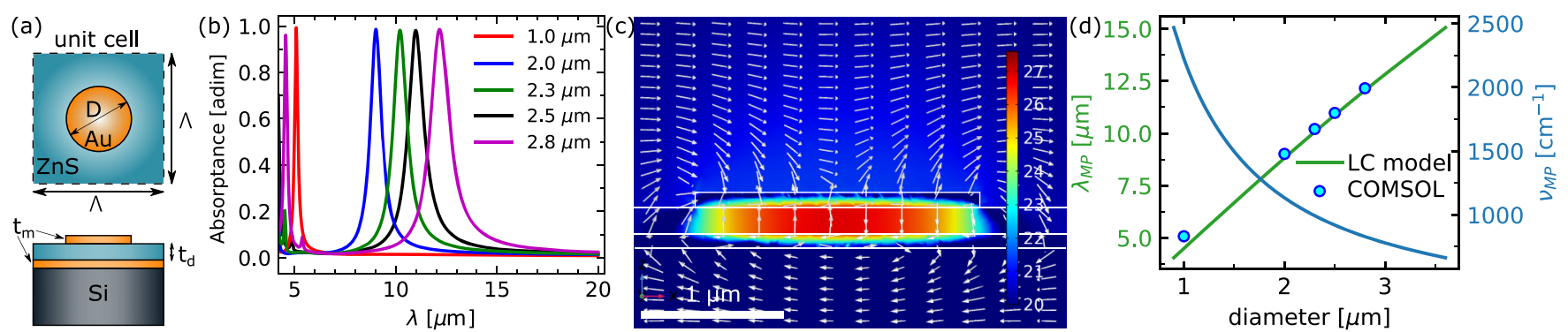

Figure 1. (a) Top view and side view of the unit cell of a $\mathrm{Au}-\mathrm{ZnS}-\mathrm{Au}$ periodic array structure on a Si substrate. Dashed lines indicate periodic boundaries. (b) Computed absorptance spectra for different Au disc diameters and equal unit cell pitch $\Lambda=4 \mu \mathrm{m}$. (c) Magnetic field intensity (logl $\left.H_{\mathrm{y}}{ }^{2}\right)$ localization around a disc of $2 \mu \mathrm{m}$ diameter. Arrows show the distribution of the electric displacement field. (d) Comparison between the magnetic polariton resonance wavelength $\lambda_{\mathrm{MP}}$ obtained from the computed spectra in (b) and $\lambda_{\mathrm{MP}}$ predicted with the LC-model.

to $14 \mu \mathrm{m}$, referred to as the "sky window", is relevant in this context because the atmosphere is IR-transparent in this range. Passive cooling devices largely utilized (structured) dielectric materials to adjust the optical properties in the mid-IR range. Nocturnal passive radiative cooling has been accomplished using a $\sim 1 \mu$ m-thick $\mathrm{SiO}$ film on an opaque $\mathrm{Al}$ layer deposited on a glass substrate. Excitation of surface phonon modes in $\mathrm{SiO}$ leads to high absorptance within the sky window. ${ }^{7,10}$ In the case of daytime passive radiative cooling, ${ }^{7,11-16}$ randomly packed micron-sized $\mathrm{SiO}_{2}$ spheres have shown good performance. They minimize the photon transport mean free path of incident solar radiation (which maximizes the scattering of solar radiation), while enhancing thermal emission in the atmospheric transparency window. ${ }^{13}$ Moreover, a remarkable example can be found in nature: the Saharan silver ant ( Cataglyphis bombycina) thermoregulatory system consists of a dense array of triangular prismatic hairs. This allows the ant to keep its body temperature below the critical maximum (53.6 ${ }^{\circ} \mathrm{C}$ ) by reflecting between 50 and $80 \%$ of the incident solar radiation (up to the NIR) and emitting heat to the surrounding environment in the mid-IR. ${ }^{4}$

Tuning of the absorption properties within this sky-window range, however, is limited when working with dielectric structures. MDM architectures, on the contrary, offer a variety of tunable parameters, allowing a specific modification of the excited resonances. For example, periodic arrays of MDM metamaterials have shown to be versatile for tuning the IR absorption peak (magnetic polariton resonance of the first order) into different regimes of the EM spectrum using the appropriate absorber dimensions. ${ }^{1,17-19}$ Likewise, the intensity of plasmonic resonances highly depends on the incidence angle, decaying drastically as the incidence angle increases with respect to normal incidence $\left(0^{\circ}\right)$. In contrast, the intensity of magnetic polariton resonances remains almost constant for incidence angles up to $\sim 60^{\circ}$ with respect to normal incidence. ${ }^{17,20}$ Additionally, several physical characteristics associated with the strong absorptance exhibited by MDM structures have been widely studied in the literature. ${ }^{9,21-25}$ Accordingly, it has been recently shown by near-field and farfield spectroscopy that a nanopatch antenna is very effective in terms of EM confinement, ${ }^{9,21,22}$ that is, the field decays very fast outside the nanopatch antenna and, therefore, the resonance condition of neighboring nanoantennas is (in general) superimposed on the optical response of the unit cell for periodic arrays. In this case, the resonant wavelength is determined by the geometric shape of the nanopatch and the dielectric height but is independent of the periodicity. ${ }^{22,23}$
In this work, we show, experimentally, that magnetic polariton resonances of the first order, induced in MDM metasurfaces, can be tailored to induce a strong absorptance peak in the mid-IR. We accomplish this with a $2 \mathrm{D}$ periodic array composed of an Au disc centered in a squared unit cell on top of a $\mathrm{ZnS}-\mathrm{Au}-\mathrm{Si}$ substrate, for the adequate $\mathrm{Au}$ disc diameter. The structures are fabricated by photolithography and characterized by IR spectroscopy. We support the experimental findings with FEM simulations and a simplified inductance-capacitance (LC) model. Furthermore, we evidence that the individual resonators can be combined in a specific manner to realize broadband absorption properties in defined spectral ranges. The obtained results can be applied to different regions of the EM spectrum by appropriate structural design. Furthermore, these results suggest a simple way to design broadband absorptance filters.

\section{MATERIALS AND METHODS}

2.1. Numerical Simulations. We simulated a $2 \mathrm{D}$ periodic array of $\mathrm{Au}$ discs on top of a $\mathrm{ZnS}-\mathrm{Au}-\mathrm{Si}$ (top to bottom) substrate. Figure la shows a diagram of a unit cell of the investigated structure. A 3D model of such a unit cell is constructed in COMSOLMultiphysics v5.4. The wave optics module is used to compute the corresponding transmittance $(T)$ and reflectance $(R)$ spectra for different disc diameters. The absorptance spectra are obtained from the energy conservation principle as $A=1-R-T$ at each wavelength. ${ }^{26}$ All simulations are performed considering normal incidence of transverse magnetic EM waves. Because of the rotational symmetry of the unit cell, the same spectra are expected for transverse electric incident waves. ${ }^{27}$ This fact has been confirmed with additional COMSOL simulations. An input port is set above the top of the Au disc at a distance of around 1.5 times the period of the unit cell. The space in between the MDM structure and the input port is filled with air. From this port, the incidence of radiation is incoming to the MDM unit cell, and also, the reflectance spectra are evaluated here. Similarly, an output port is set at the back of the Si substrate. On this port, the transmittance spectra are evaluated. Details about the computation of $T$ and $R$ are given in the Supporting Information. Additionally, two perfectly matched layers are set: one right above the input port and another one right below the output port. All four lateral boundaries are set as Bloch-Floquet periodic boundaries. The maximum mesh size is $\sim 1 \mu \mathrm{m}$ in the air domain, and the minimum mesh size is $\sim 20$ $\mathrm{nm}$ in the Au components. The complex dielectric function of $\mathrm{Au}$ is computed based on the Drude-Lorentz model, ${ }^{28}$ and for $\mathrm{ZnS}$, it is taken from Querry. ${ }^{29}$ Further details about the boundary conditions and the setup for the numerical simulations are presented in the Supporting Information.

Optical nanocircuits have been shown to be reliable for predicting certain optical properties of nano- to microstructured materials. ${ }^{30}$ Accordingly, we have used an elementary inductor-capacitor circuit (LC model) to compute the first magnetic polariton resonance in the 
$\mathrm{Au}-\mathrm{ZnS}-\mathrm{Au}$ structures as a function of the diameter of the $\mathrm{Au}$ discs. ${ }^{31}$ This model simulates the metallic components (Au disc and $\mathrm{Au}$ film) as inductors composed of the mutual inductance of parallel plates and from the contribution of drift electrons. These components are linked by the capacitance generated between them and the dielectric layer. Additionally, a parallel plate capacitance is introduced to take into account the gap capacitance between neighboring discs. Full details about the LC model are provided in the Supporting Information.

2.2. Sample Fabrication. The fabrication of $2 \mathrm{D}$ periodic arrays of $\mathrm{Au}$ discs on top of a $\mathrm{ZnS}-\mathrm{Au}-\mathrm{Si}$ substrate is performed following a bottom-to-top approach. Figure 1a shows the unit cell of such a structure for a single-diameter configuration. The fabrication of samples in the multiple-diameter configuration is conducted in the same way, that is, $2 \mathrm{D}$ periodic arrays of Au discs of multiple diameters on top of a $\mathrm{ZnS}-\mathrm{Au}-\mathrm{Si}$ substrate are also fabricated.

A $280 \mu \mathrm{m}$-thick $\mathrm{Si}$ wafer CZ-(100)-Test grade from MicroChemicals $\mathrm{GmbH}$ is used as the supporting substrate. It is coated with $\mathrm{Au}$ and $\mathrm{ZnS}$ films of 100 and $200 \mathrm{~nm}$ thicknesses, respectively. Both layers are obtained by physical vapor deposition (PVD) using a BALZERS BA510 batch coating system. Subsequently, a double-layer lift-off process is used to produce a $2 \mathrm{D}$ periodic array of microholes on the photoresist bilayer: first, a positive lift-off photoresist (LOR $3 \mathrm{~B}$, purchased from micro resist technology $\mathrm{GmbH}$ ) is spin-coated at $3000 \mathrm{rpm}$ for $45 \mathrm{~s}$ using an acceleration rate of $100 \mathrm{rpm} / \mathrm{s}$. Immediately, the substrate is soft-baked on a hot plate at $170{ }^{\circ} \mathrm{C}$ for 5 min. Second, a positive photoresist (MICROPOSITS1813, purchased from micro resist technology $\mathrm{GmbH}$ ) is spin-coated on top of the previous LOR 3B layer at $5000 \mathrm{rpm}$ for $45 \mathrm{~s}$ using an acceleration rate of $100 \mathrm{rpm} / \mathrm{s}$. Afterward, the structure is soft-baked on a hot plate at $100{ }^{\circ} \mathrm{C}$ for $60 \mathrm{~s}$. The photoresists are exposed to a UV source $(385$ $\mathrm{nm}$ wavelength) using a MicroWriter ML3 Baby Plus from Durham Magneto Optics Ltd, UK. The exposure dose is set to $420 \mathrm{~mJ} \mathrm{~cm}{ }^{-2}$. The written structures (2D periodic disc arrays) are developed by immersing the sample in a developer solution (MICROPOSITMF319, purchased from micro resist technology $\mathrm{GmbH}$ ) and gently stirring it for $30 \mathrm{~s}$. Right after, it is again stirred in ultrapure water for $30 \mathrm{~s}$ and finally dried with compressed air. Afterward, an Au layer of $100 \mathrm{~nm}$ thickness is evaporated on the photoresist frame using PVD. The evaporated $\mathrm{Au}$ fills the microholes of the bilayer photoresist frame. The unwanted photoresist-Au frame is removed by placing the sample in 1-methyl-2-pyrrolidinone (purchased from SigmaAldrich) for $20 \mathrm{~min}$. Then, it is ultrasonically cleaned at $80 \mathrm{kHz}$ for 5 min three times. Later, the sample is placed in ethanol absolute (VWR chemicals) and is ultrasonically cleaned for $5 \mathrm{~min}$. Finally, it is dried with compressed air. More details about the fabrication process, including a flowchart, are presented in the Supporting Information.

2.3. Optical Characterization. A Fourier transform infrared (FTIR) microscope (LUMOS from Brucker) is used to obtain the absorptance spectra of the samples. Reflectance spectra are measured directly in the reflection configuration, and the corresponding absorptance spectra are obtained afterward using the energy conservation principle: $A=1-R$, as mentioned above. Transmittance is nearly zero in the studied spectral range of interest for the samples analyzed in this work. The optically thick Au layer $(100 \mathrm{~nm})$ prevents transmission, as confirmed by numerical simulations (see the Supporting Information) and literature reports. ${ }^{8,23,32}$ Accordingly, it is neglected $(T=0)$ in the experimental analysis. Measurements are performed covering the range from 450 to $4000 \mathrm{~cm}^{-1}$ with $4 \mathrm{~cm}^{-1}$ spectral resolution; each spectrum is the result of averaging 64 subsequent acquisitions. The FTIR microscope is equipped with an $8 \times$ objective (NA 0.6), and each acquisition is obtained from the average signal collected from a $500 \mu \mathrm{m} \times 500 \mu \mathrm{m}$ area on the sample. Unpolarized IR radiation is used as incident excitation. An unpatterned zone of the same sample is used as a reference, that is, a $500 \mu \mathrm{m} \times 500 \mu \mathrm{m}$ area of $\mathrm{ZnS}-\mathrm{Au}$ on the $\mathrm{Si}$ wafer. This choice is used to avoid baseline offsets.

2.4. Structural Characterization. Scanning electron microscopy (SEM) images are obtained with an LEO 1530 Gemini field emission scanning electron microscope (Carl Zeiss AG, Oberkochen,
Germany). The In Lense detector is used for imaging all samples, and the gun's acceleration voltage is set between 2 and $3 \mathrm{kV}$.

Atomic force microscopy (AFM) images are recorded using a Nanoscope Dimension V (Veeco, USA) instrument with a Dimension 3100 controller. The instrument is operated in the tapping mode with OTESPA-R3 cantilevers (Bruker). The resulting images are evaluated with NanoScope Analysis software. Moreover, a slope analysis was performed on each sample to verify the sharpness of the fabricated $\mathrm{Au}$ discs. Details of the slope analysis are presented in the Supporting Information.

Additionally, 3D reconstructed optical images taken with a laser scanning microscope (Olympus, LEXT) are used to show the uniformity of the samples regarding the periodicity and geometry of the discs. This laser scanning microscope allows a fast qualitative evaluation of the fabricated samples. A $100 \times$ objective was used with N.A. 0.95 and a z-scanning pitch of $120 \mathrm{~nm}$. Additionally, a 1.3X digital zoom was used to obtain a better view of the Au discs imaged.

\section{RESULTS AND DISCUSSION}

3.1. Magnetic Polariton Absorption. We first explore the fundamental influence of the disc diameter on the absorptance spectrum of monomodal arrays of Au discs by numerical simulations. Figure 1a shows the unit cell of such a 2D periodic structure.

Figure $1 \mathrm{~b}$ summarizes the absorptance spectra obtained from FEM simulations, corresponding to an Au disc of 1.0, 2.0, 2.3, 2.5, and $2.8 \mu \mathrm{m}$ diameter centered in the unit cell. The pitch is set to $\Lambda=4 \mu \mathrm{m}$ for these simulations. The disc diameter is tailored to promote absorptance peaks within the sky window $(7.5-14 \mu \mathrm{m})$. Each absorptance spectrum shows a strong, single absorptance peak as the Au disc diameter is increased from 1.0 to $2.5 \mu \mathrm{m}$ in the unit cell. A spectral range from 4 to $20 \mu \mathrm{m}$ is considered. The absorptance peak shifts to longer wavelengths as the $\mathrm{Au}$ disc diameter increases. This trend is observed for all analyzed disc diameters, as shown in Figure $1 \mathrm{~d}$. Furthermore, for Au discs of $2.8 \mu \mathrm{m}$ diameter, absorptance peaks at wavelengths around 12 and $4.5 \mu \mathrm{m}$ are obtained (see Figure 1b).

Magnetic polaritons are regarded as resonances with magnetic response coupled to a nonmagnetic material. ${ }^{17,18}$ Accordingly, the origin of the strong absorptance obtained can be explained by looking at the cross section of the EM field distribution at the central wavelength of each peak. Figure 1c shows an exemplary cross section of the EM field distribution (at the peak wavelength $\lambda=8.99 \mu \mathrm{m}$ ) on a unit cell with an $\mathrm{Au}$ disc of $2.0 \mu \mathrm{m}$ diameter. The electric displacement field is represented by arrows, whose tails are normalized with respect to the incident EM field. The antiparallel orientation of the displacement field in the $\mathrm{ZnS}$ layer denotes that current densities of the opposite direction occur in the metallic components (Au disc and Au film). In agreement with Lenz's law, these antiparallel currents activate a diamagnetic effect inside the $\mathrm{ZnS}$ layer. To illustrate this, the corresponding cross section of the normalized magnetic field intensity $\left(\log \mid H_{\mathrm{y}}{ }^{2}\right)$ is also shown in Figure 1c. The color bar indicates the intensity of the magnetic field normalized with respect to that of the incident field. The magnetic field in the ZnS layer is clearly confined in a cylindrical domain of diameter around the size of the $\mathrm{Au}$ disc mentioned above and height almost equal to the thickness of the $\mathrm{ZnS}$ layer. In the surroundings (outside) of these regions, the magnetic field intensity drops about five orders of magnitude relative to its intensity in the localized domain. This localization of the magnetic field is the reason for the resonant absorptance peak observed in the spectra and can 


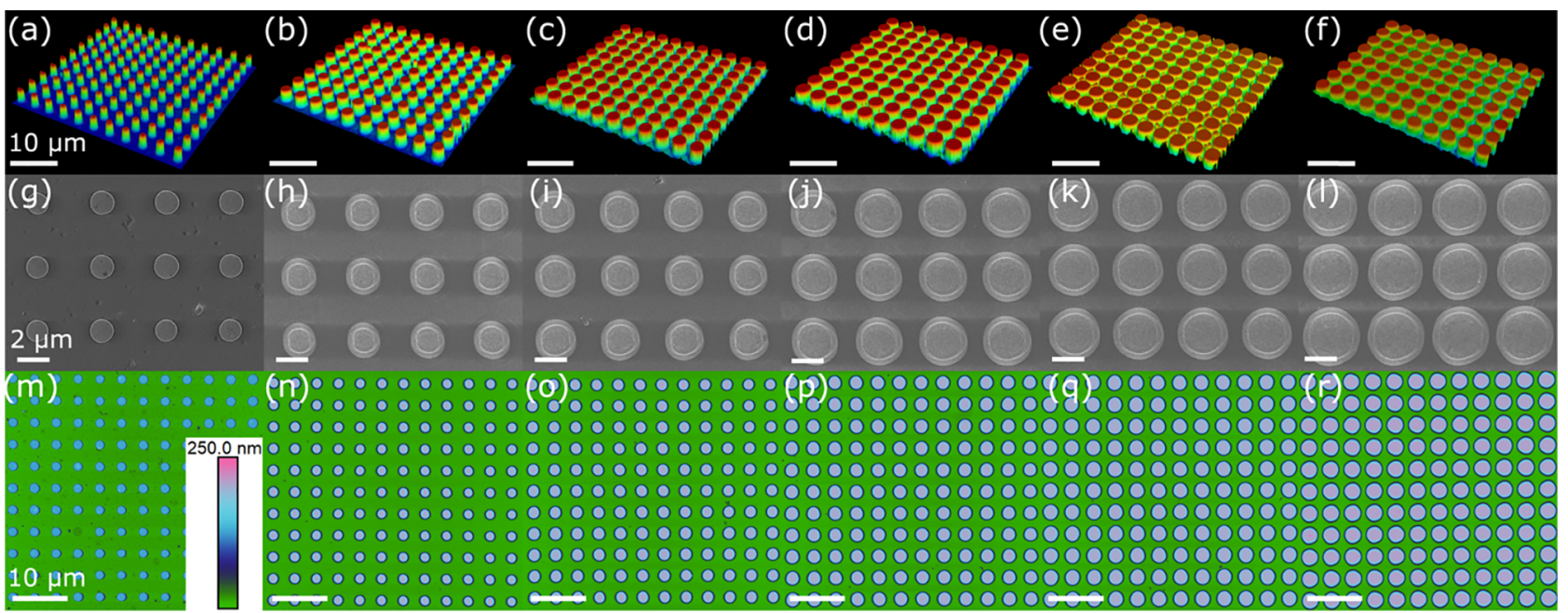

Figure 2. (a-g) Exemplary laser scanning microscopy images of the different fabricated samples. The diameters of the Au discs are (a) 1.57, (b) 1.80 , (c) 2.25, (d) 2.65, (e) 2.91, (f) 3.26, and (g) $3.53 \mu \mathrm{m}$. ( $\mathrm{h}-\mathrm{n}$ ) SEM images over a few periods of the samples. (o-u) AFM images of the samples; the colorbar indicates the height of the sample.

be attributed to the first resonant mode of the magnetic polariton. Nevertheless, depending on the size of the Au disc, also higher-order MP modes can be excited, such as the thirdorder mode observed at around $4.5 \mu \mathrm{m}$ for the $2.8 \mu \mathrm{m}$ disc (see Figures $1 \mathrm{~b}$ and $\mathrm{S} 2$ in the Supporting Information).

The resonance wavelength of the magnetic polariton $\lambda_{\mathrm{MP}}$ is extracted from each computed absorptance spectrum as the central wavelength of the corresponding absorptance peak. Figure 1d shows these results (represented by dots) as a function of the corresponding Au disc diameter. Additionally, the LC model provides a fast and accurate way to estimate the frequency corresponding to the first resonance of the magnetic polaritons. These results are plotted on the right axis of Figure $1 \mathrm{~d}$ in wavenumber units. Afterward, the corresponding resonant wavelengths are computed using the relation $\lambda_{\mathrm{MP}}=$ $1 / \nu_{\mathrm{MP}}$, where $\nu_{\mathrm{MP}}$ is the wavenumber resonance of the magnetic polariton. The results obtained for $\lambda_{\mathrm{MP}}$ as a function of the disc diameter are shown in Figure 1d using a continuous green line. Both methods (LC model and peak evaluation from FEM simulation) provide consistent results for $\lambda_{\mathrm{MP}}$. The parameters used for the LC model are specified in the Supporting Information. Overall, the adjustment of the disc diameter controls the resonant absorption of magnetic polaritons and, consequently, allows tuning the mid-IR absorption of such MDM structures.

We now turn to the experimental realization of such structures. Optical inspection of the fabricated 2D periodic arrays of $\mathrm{Au}$ discs on $\mathrm{ZnS}-\mathrm{Au}-\mathrm{Si}$ substrates is performed with the LEXT microscope. Figure $2 \mathrm{a}-\mathrm{g}$ shows large areas of the fabricated samples with different Au disc diameters. The period of the unit cell is fixed to $4 \mu \mathrm{m}$ for all cases. Consequently, the $\mathrm{Au}$ discs grow closer to each other as their size increases. The $\mathrm{Au}$ disc diameter characteristic for each sample is obtained by analyzing SEM images; around 130 discs were counted for each sample, and the average is reported. Moreover, SEM images shown in Figure $2 \mathrm{~h}-\mathrm{n}$ display a few unit cells of each sample. It confirms that each unit cell consists of a centered $\mathrm{Au}$ disc of a given diameter, and the period is fixed. The diameters of the $\mathrm{Au}$ discs increase monotonically, as already observed by the optical images. Complementary AFM images are performed on the samples to further investigate the diameter and the height of the discs (Figure 2o-u). The same height scale (color bar) is used in all cases ranging from 0 to $250 \mathrm{~nm}$. From these images, the uniformity of the $2 \mathrm{D}$ periodic arrays of $\mathrm{Au}$ discs is confirmed, for all seven diameters. The average height of the Au discs on top of the $\mathrm{ZnS}$ layer is about $193 \mathrm{~nm}$. Furthermore, a slope analysis (see the Supporting Information) shows that the slopes of the $\mathrm{Au}$ disc profiles of the fabricated single diameter samples are $\sim 530=89.89^{\circ}$. Therefore, the Au disc profiles are sharp enough and should not be considered as truncated solids.

Absorptance spectra of the samples are obtained from FTIR microscopy. Reflectance is directly measured using a LUMOS microscope, and absorptance is obtained from the energy conservation principle, as described in the optical characterization Section. Figure 3a shows the results for all fabricated, monomodal samples. Each spectrum (continuous line) is obtained from the average of 5-12 inspected areas at different positions of the sample. Each measurement is carried out on a $500 \mu \mathrm{m} \times 500 \mu \mathrm{m}$ area that includes around 15,625 unit cells, that is, between 78,125 and 187,500 unit cells are analyzed. The standard error computed from these measurements is displayed as a shadow (lighter color) of the continuous line. The MP resonance shifts to longer wavelengths, as the diameters of the $\mathrm{Au}$ discs increase. The spectra are color-coded to distinguish the different Au disc diameters: $1.57 \mu \mathrm{m}$ (blue), $1.80 \mu \mathrm{m}$ (gray), $2.25 \mu \mathrm{m}$ (green), $2.65 \mu \mathrm{m}$ (red), $2.91 \mu \mathrm{m}$ (dark orange), $3.26 \mu \mathrm{m}$ (indigo), and $3.53 \mu \mathrm{m}$ (cyan). The full width at half-maximum (FWHM) of the spectra is not constant. This is in agreement with the fact that different disc diameters promote different (and independent) magnetic polariton resonances. However, the FWHM is comparable between samples with the largest disc diameters (see Figure S4a in the Supporting Information).

Magnetic polariton resonances of the first order are extracted from the average absorptance spectra (continuous lines) of Figure 3a. The resonant wavelengths of the MP $\left(\lambda_{\mathrm{MP}}\right)$ are obtained as the central wavelength of the strong absorptance peak in each spectrum. Figure $3 b$ shows the results of $\lambda_{\mathrm{MP}}$ as a function of the Au disc diameters in the fabricated samples. Scatters feature the same color code as in the corresponding spectra. A monotonic increase in the 

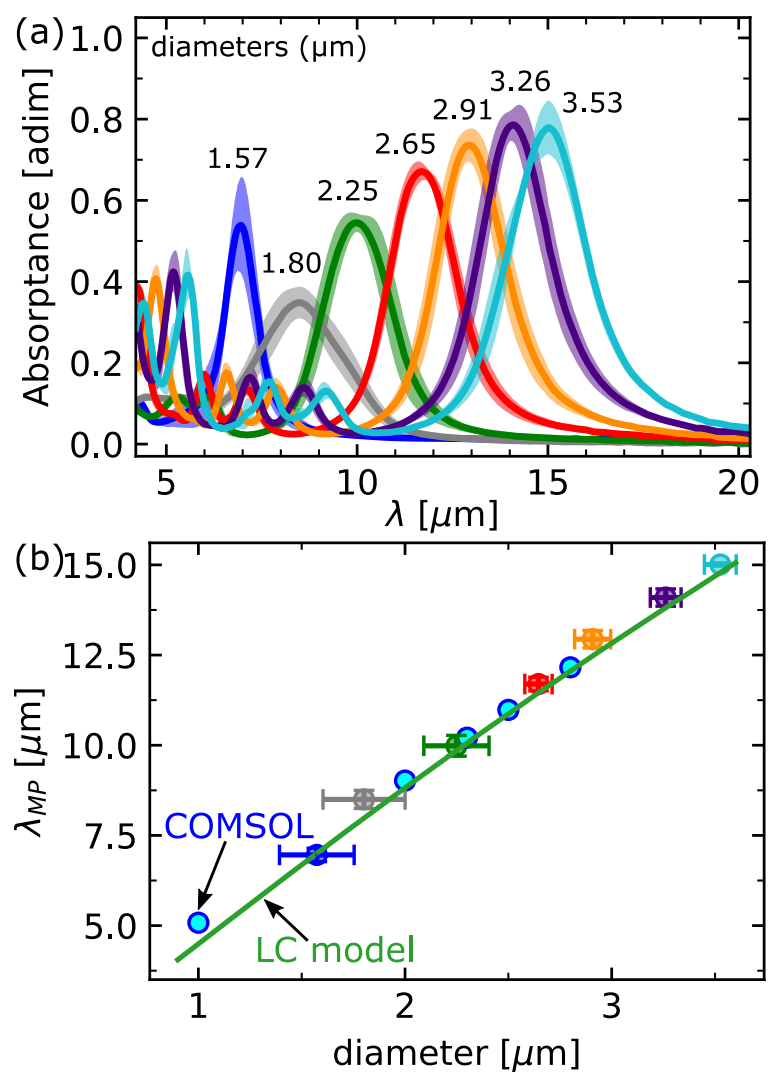

Figure 3. (a) Absorptance spectra measured for each sample showed in Figure 2; the lighter colors show the standard deviation from different measured spectra. (b) Resonance wavelength of the magnetic polariton $\lambda_{\mathrm{MP}}$ as a function of the disc diameter. Circles are color-coded as their corresponding spectra in (a). The green line corresponds to the prediction obtained using the LC model. Cyanfilled circles are results from COMSOL simulations. Error bars are the standard deviations computed from the different measured spectra and from SEM images. resonant wavelength $\lambda_{\mathrm{MP}}$, in the spectral range $\sim 7-15 \mu \mathrm{m}$, is obtained by increasing the Au disc diameters from 1.57 to 3.53 $\mu \mathrm{m}$. The experimental results are compared with the prediction obtained from the LC model. The predicted resonant wavelength $\lambda_{\mathrm{MP}}$ as a function of the $\mathrm{Au}$ disc diameter in a unit cell is represented by the green line in Figure $3 \mathrm{~b}$ and is equal to that shown in Figure 1d, that is, the same parameters are used. Good agreement is found between the experimental results and the LC model prediction. This confirms the utility and versatility of this elementary LC circuit for the design of optimum geometrical dimensions of $2 \mathrm{D}$ arrays of $\mathrm{Au}$ discs on a $\mathrm{ZnS}-\mathrm{Au}-\mathrm{Si}$ substrate for the desired MP resonance in the mid-IR spectral range. Moreover, this simple model is also applicable to similar MDM periodic structures and can pave the way to explore other spectral regimes of the EM spectrum. Nevertheless, the LC model slightly underestimates the resonant wavelengths of the MP in some cases, particularly for the larger Au discs.

3.2. Multimodal Magnetic Polariton Absorption. We now want to use this set of individually absorbing discs to design a more broadband absorption within the sky-window range. Figure $4 \mathrm{a}-\mathrm{d}$ shows exemplary laser scanning microscopy images of the double-diameter samples. In this case, the unit cell for each sample contains four Au discs of two different diameters $D_{1}$ and $D_{2}$, with $D_{1}<D_{2}$. The following configurations are studied: $D_{1}=1.85 \mu \mathrm{m}$ and $D_{2}=2.50 \mu \mathrm{m}$ (Figure 2a), $D_{1}=2.19 \mu \mathrm{m}$ and $D_{2}=2.72 \mu \mathrm{m}$ (Figure 2b), $D_{1}=$ $2.27 \mu \mathrm{m}$ and $D_{2}=2.77 \mu \mathrm{m}$ (Figure $2 \mathrm{c}$ ), and $D_{1}=2.38 \mu \mathrm{m}$ and $D_{2}=2.86 \mu \mathrm{m}$ (Figure $2 \mathrm{~d}$ ). The periodicity of the $2 \mathrm{D}$ arrays is 8 $\mu \mathrm{m} \times 8 \mu \mathrm{m}$ for these samples. These configurations of discs diameters are chosen to tailor the absorptance within the sky window, based on results from Figure $3 \mathrm{~b}$. The four Au discs in each unit cell are arranged such that discs with equal diameters lay along the diagonals, as shown by the SEM images in Figure $4 \mathrm{e}-\mathrm{h}$. This choice is favored to procure polarizationindependent absorptance in these $2 \mathrm{D}$ structures. The Au disc diameter $\left(D_{1}\right.$ and $\left.D_{2}\right)$ characteristics of each sample are
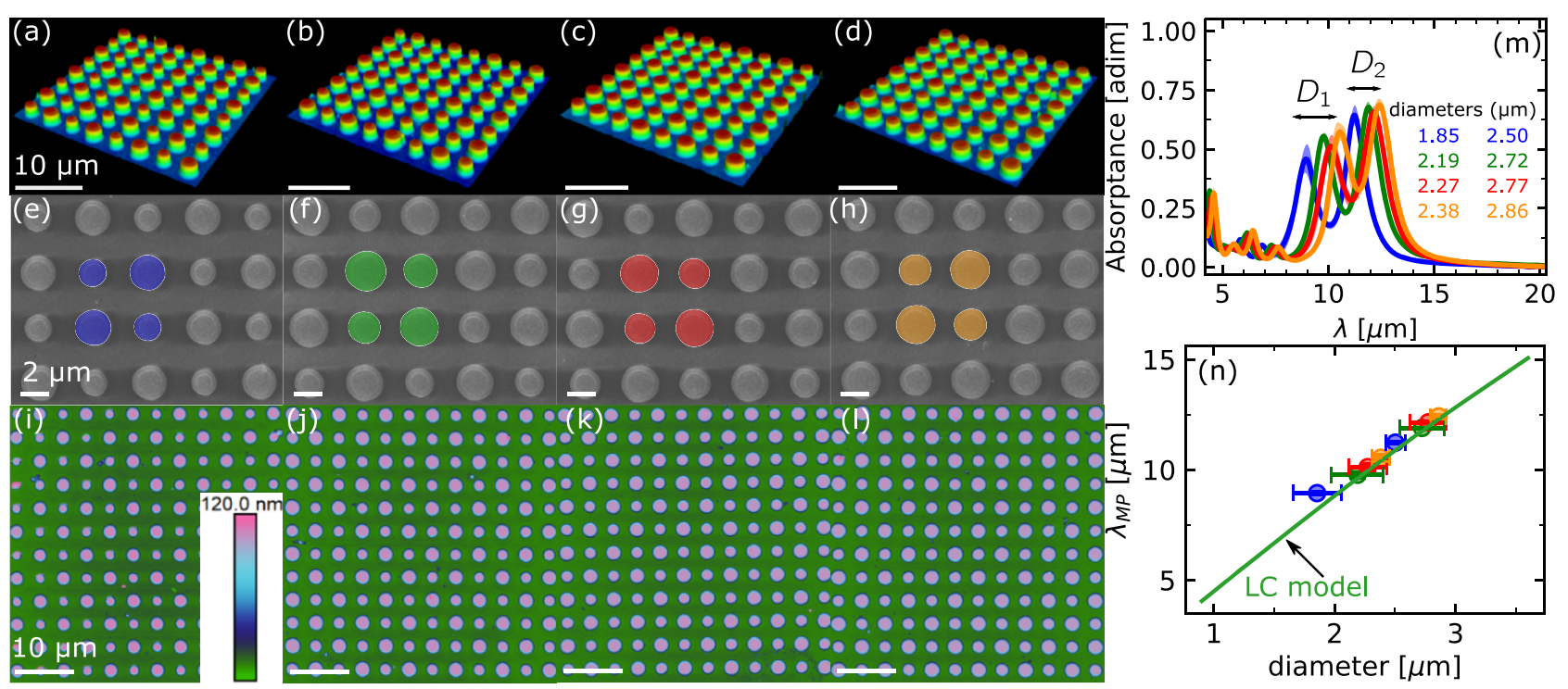

Figure 4. $(\mathrm{a}-\mathrm{d})$ Exemplary laser scanning microscopy images of the double-diameter samples. $(\mathrm{e}-\mathrm{h})$ SEM images over a few periods of these samples. (i-l) AFM images of the double-diameter samples, the color bar ranges from 0 to $120 \mathrm{~nm}$ and indicates the height of the sample. (m) Absorptance spectra of these samples. The spectra are color-coded according to the SEM images. (n) Magnetic polariton resonance wavelength $\lambda_{\mathrm{MP}}$ as a function of the diameters of the Au discs in each unit cell. Error bars are the standard deviations computed from the different spectra and from SEM images. The continuous line shows the results predicted by the LC model. 

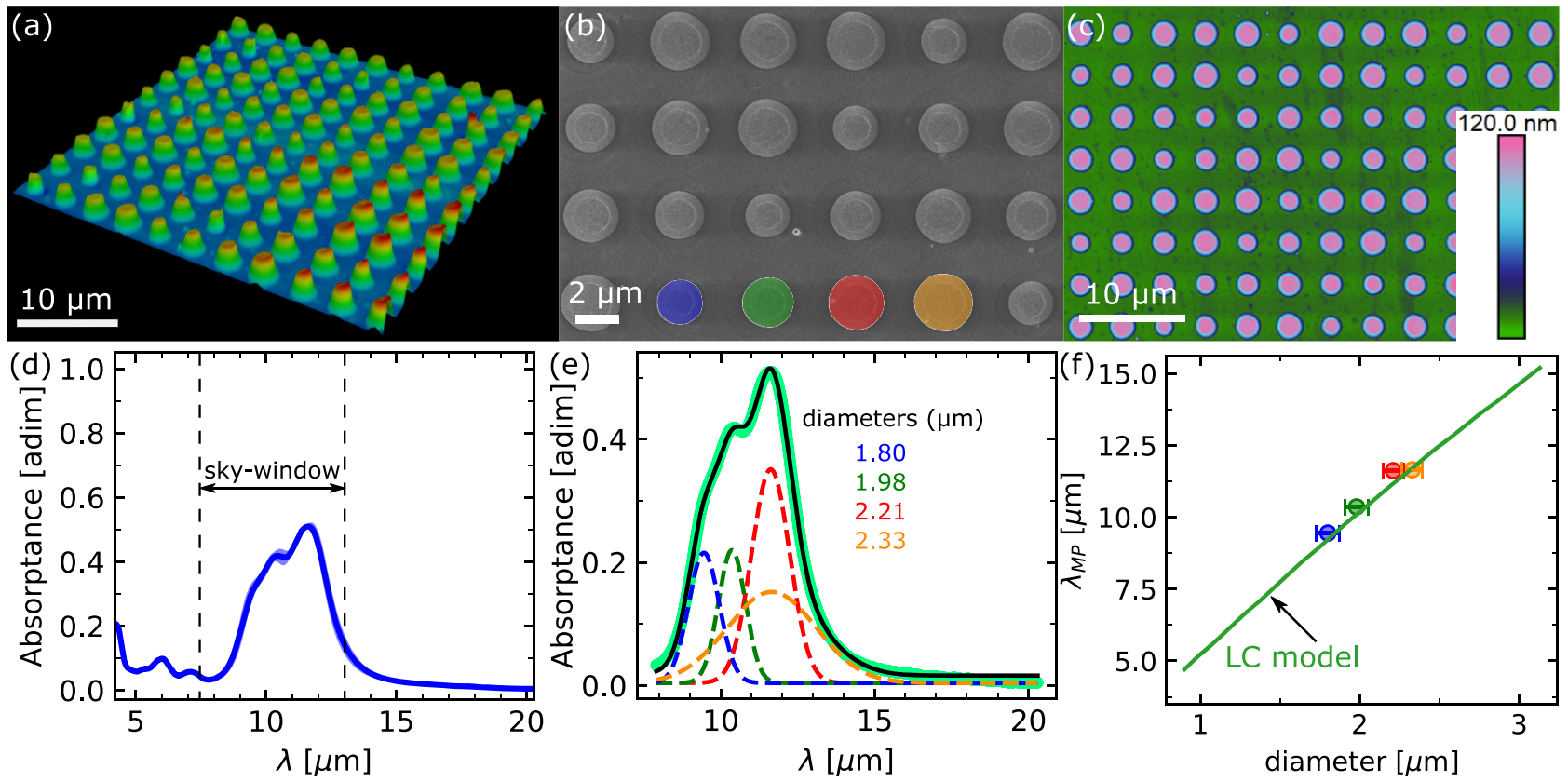

Figure 5. (a) Exemplary laser scanning microscopy image of a quadruple-diameter sample. (b) SEM image over a few periods of this sample. The four different diameters are color-coded in the horizontal and vertical directions. (c) AFM image of the sample. The color bar ranges from 0 to 120 $\mathrm{nm}$ and indicates the height of the Au discs. (d) Absorptance spectra of this sample. A strong broadband absorptance is observed within the sky window. (e) Average absorptance spectra and its decomposition into four absorptance peaks corresponding to the four different disc diameters. (f) Magnetic polariton resonances (obtained from (e)) as a function of the measured Au disc diameters. Error bars are the standard deviations of the diameters obtained from SEM images. The continuous line shows the results predicted by the LC model.

obtained from analyzing SEM images; 110 to 270 discs were counted for each sample and the average is reported. Additionally, AFM images (Figure 4i-l) are used to show the uniformity of $2 \mathrm{D}$ arrays over several periods and to measure the $93 \mathrm{~nm}$ average height of the Au discs, which were obtained by PVD. Furthermore, a slope analysis (see the Supporting Information) shows that the slopes of the Au disc profiles of the fabricated double-diameter samples are $\sim 350=$ $89.84^{\circ}$. Therefore, the $\mathrm{Au}$ disc profiles are sharp enough and should not be considered as truncated solids.

Figure $4 \mathrm{~m}$ shows the absorptance spectra obtained for the described samples. Each spectrum (continuous line) is obtained from the average of 5-25 probed zones at different positions in the sample. Each measurement is carried out on a $500 \mu \mathrm{m} \times 500 \mu \mathrm{m}$ area that includes around 3096 unit cells, that is, between 19,530 and 97,650 unit cells are analyzed. In this case, all spectra show two strong absorptance peaks instead of a single one (compare with Figure 3a). Moreover, each absorptance peak shifts to longer wavelengths, as the diameters of the $\mathrm{Au}$ discs increase. Peak resonant wavelengths $\left(\lambda_{\mathrm{MP}}\right)$ are extracted from each spectrum and plotted as a function of the diameter. These resonant wavelengths are obtained as the central wavelength of each strong absorptance peak in every spectrum. The wavelength corresponding to the first strong absorptance peak (shorter wavelength) is paired to $D_{1}$, and the second one (longer wavelength) is paired to $D_{2}$ in each spectrum. Figure $4 \mathrm{n}$ shows these results: scatters of the same color indicate that the resonant wavelengths are obtained from the same spectra. The same color code as in the SEM images and Figure $4 \mathrm{~m}$ is used here. A monotonic increase in resonant wavelength $\lambda_{\mathrm{MP}}$, in the spectral range $\sim 8.9-12.4 \mu \mathrm{m}$, is obtained for the studied configurations. Additionally, the prediction of the MP resonances of the first order as a function of the $\mathrm{Au}$ diameter is plotted as a continuous green line. This simulation is equal to the one presented in Figure $3 \mathrm{~b}$. The excellent agreement between the experimental results (scatters) and the LC model (continuous line) suggests that both absorptance peaks can be attributed to individual magnetic polariton resonances of the first order of the respective disc. The overall absorption of the bimodal array can, consequently, be understood as a superposition of both resonances. Moreover, an analysis of the FWHM on these samples is presented in the Supporting Information. It shows that the FWHM of the MP resonances decreases as the diameter increases. A comparison to the FWHM of the peaks in the case of monomodal disc arrays (Figure S4a) shows a slightly decreased FWHM. Besides this, there is no hint of EM or magnetic coupling between the discs. Particularly, the peak positions can be regarded as a linear combination of the two underlying disc sizes. Our measurements, thereby, confirm the known behavior of nanoantennas in the mid-IR range, where a strong field confinement within the structure has been experimentally and theoretically confirmed. The fast decay outside the structure prevents the coupling between adjacent resonators in such systems. ${ }^{9,22,23,25}$

We take this concept further and now utilize four individual discs to fully address the sky-window range. In accordance with the previous results for single- and double-diameter configurations, each unit cell should be composed of $16 \mathrm{Au}$ discs of four different diameters. Broadband absorptance within the sky window is actually achieved with the following $\mathrm{Au}$ disc diameters: $D_{1}=1.80 \mu \mathrm{m}, D_{2}=1.98 \mu \mathrm{m}, D_{3}=2.21 \mu \mathrm{m}$, and $D_{4}$ $=2.33 \mu \mathrm{m}$, determined from SEM images. Figure $5 \mathrm{a}$ shows an exemplary laser scanning microscopy image taken from a quadruple-diameter sample. The $16 \mathrm{Au}$ discs are arranged in a $16 \mu \mathrm{m} \times 16 \mu \mathrm{m}$ unit cell, as shown in Figure $5 \mathrm{~b}$. This SEM image shows the different diameters involved in different colors; the details of this unit cell are provided in the 
Supporting Information. The Au disc diameters increase from the left to the right and from the bottom to the top in each unit cell. The main diagonal of a unit cell consists of the Au disc of a minimum diameter. Optical microscopy, SEM, and AFM confirm the uniformity of this sample as well, which comprises a height of the $\mathrm{Au}$ discs of $96 \mathrm{~nm}$. Furthermore, the slope analysis (see the Supporting Information) shows that the slopes of the $\mathrm{Au}$ disc profiles of the fabricated quadruple diameter are $\sim 440=89.87^{\circ}$. Figure $5 \mathrm{~d}$ shows an exemplary absorptance spectrum (continuous line) obtained from an average of 12 different probed zones in the sample. Each measurement comprehends a $500 \mu \mathrm{m} \times 500 \mu \mathrm{m}$ area that includes around 976 unit cells, that is, at least 11,712 are analyzed. The standard error is computed from the different probed zones and is displayed as a shadow (lighter color) of the continuous line. A strong broadband absorptance is observed instead of four distinguishable absorptance peaks. This can be explained by a similar size of the Au discs in the unit cell, combined with the spectral proximity between the induced MP resonances. ${ }^{25}$ Accordingly, the broadband spectra are fitted to four Gaussian curves representing independent $\mathrm{MP}$ resonances because of the four different $\mathrm{Au}$ discs. Figure 5e shows the obtained results, the light green continuous line displays the average spectra from measurements, and the black line represents the fit (sum of four Gaussian curves). The Gaussian functions are plotted in dashed lines using the same color code as in Figure 5b. This analysis is also valid because the $\mathrm{Au}$ structures are not truncated solids such as cones or pyramids, which have been previously applied to induce absorptance bands. ${ }^{7,33}$ Figure $5 \mathrm{f}$ shows the relation between the MP resonances and the Au disc diameters. The same color code as in Figure 5e is used. In this case, no error bars are displayed on $\lambda_{\text {MP }}$ because no experimental uncertainty can be associated. The LC model (continuous green line) can predict the experimental results by taking into account that the charge distribution in a unit cell is slightly increased with respect to the single- and double-diameter configurations, because of the presence of four different $\mathrm{Au}$ discs (explanations are given in the Supporting Information). Consequently, the broadband absorption is, in this case, the result of the superposition of individual disc resonators. Considering the predictable behavior of monomodal, bimodal, and multimodal disc combinations, such MDM structures allow designing specific absorption profiles, at least in the range from 5 to $15 \mu \mathrm{m}$.

We want to put this broadband mid-IR tuning into the perspective of potential fields of interest. MDM structures are well suitable to adjust the absorption and emission properties in a wide wavelength range. Operating at mid-IR energies, such structures strongly influence the emission of thermal energy. Consequently, thermal camouflage and passive cooling applications are foreseeable devices for such structures. We provide an optical impression of the thermal emission engineering in Figure S8 (Supporting Information). Here, we compare the emissivity of our fabricated monomodal disc arrays subjected to $100{ }^{\circ} \mathrm{C}$ to moderately enhance mid-IR emission. The systematic change in measured array temperature is commensurate with the spectral changes. Solely focusing on the IR properties, however, is not sufficient, in particular, for daytime passive cooling applications. This requires a rigorous exclusion of any absorption in the visible to near IR range. The structures fabricated here, however, do absorb at least partially in the solar range, for example, because of higher-order modes of the magnetic polaritons (see Figure
S9 in the Supporting Information). Consequently, additional optical elements such as selective solar reflectors and IR transmitters are needed for an application as a passive daytime cooler. $^{34-37}$ Nevertheless, such MDM structures are good candidates for nocturnal radiative cooling. Finally, because of the fact that MP resonances depend very weakly on the period, ${ }^{1,18}$ large surfaces with similar optical absorptance characteristics compared to those presented in this work can be produced using colloidal lithography with micron-sized particle templates. However, according to Babinet's principle, the thickness of the hole mask structures must be kept thinner than their characteristic length. ${ }^{38-40}$ This provides a reasonable perspective to access micron-sized disc (or hole) arrays to cover a sufficiently large area for any conceivable usage in the macroscopic world.

\section{CONCLUSIONS}

In this work, we showed that the magnetic polariton resonance of first order, obtained from a $2 \mathrm{D}$ periodic array of an Au disc (centered in a squared unit cell) on top of a $\mathrm{ZnS}-\mathrm{Au}-\mathrm{Si}$ (top to bottom) substrate, can be tailored to induce a strong absorptance peak in the mid-IR. We characterized their mid-IR optical properties by numerical simulations using the finite element method, by a simple LC model and by experimental analysis of such a structure. Additionally, we demonstrated that such optical resonators could be used to tune the absorption properties in the mid-IR range by a simple linear combination: using a unit cell composed of four Au discs of two different diameters, it is possible to induce double-band absorptance in the mid-IR. Using a unit cell of $16 \mathrm{Au}$ discs of four different diameters induced a broadband absorption, which could be tailored to specifically span the entire sky-window range. The LC model was used to confirm that the induced magnetic polariton resonances are independent of the neighboring disc resonances in either case. We expect that this superposition approach to broadband optical tuning can be further extended beyond the spectral range investigated here ( 5 to $15 \mu \mathrm{m}$ ) as long as a strong field confinement within the optical structure is preserved.

\section{ASSOCIATED CONTENT}

\section{Supporting Information}

The Supporting Information is available free of charge at https://pubs.acs.org/doi/10.1021/acsami.0c18160.

Magnetic polariton resonances: COMSOL simulations, LC model, and FWHM, details about the fabrication process followed, details about the quadruple-diameter configuration, explanation of the AFM slopes analysis, results of the Au disc dimensions, thermographic test on the single diameter samples, and vis-NIR spectra of the samples (PDF)

\section{AUTHOR INFORMATION}

\section{Corresponding Authors}

Nelson W. Pech-May - Department of Chemistry, Physical Chemistry I, University of Bayreuth, Bayreuth 95447, Germany; Bundesanstalt für Materialforschung und -prüfung (BAM), Berlin 12200, Germany; (1) orcid.org/0000-00025822-482X; Email: nelson.pech@uni-bayreuth.de

Markus Retsch - Department of Chemistry, Physical Chemistry I and Bavarian Polymer Institute, University of 
Bayreuth, Bayreuth 95447, Germany; 이잉.org/0000-

0003-2629-8450; Email: markus.retsch@uni-bayreuth.de

\section{Author \\ Tobias Lauster - Department of Chemistry, Physical Chemistry I and Bavarian Polymer Institute, University of Bayreuth, Bayreuth 95447, Germany}

Complete contact information is available at:

https://pubs.acs.org/10.1021/acsami.0c18160

\section{Funding}

This project has received funding from the European Research Council (ERC) under the European Union's Horizon 2020 research and innovation program (grant agreement no. 714968).

\section{Notes}

The authors declare no competing financial interest.

\section{ACKNOWLEDGMENTS}

Support by the Bavarian Polymer Institute is acknowledged. N.W.P.-M. acknowledges support from an Adolf Martens fellowship at BAM Berlin and from a postdoctoral stay at the University of Bayreuth (2017-2019). We thank Kishin Matsumori and Marius Schöttle for help with additional UV/ vis reflection and thermography measurements.

\section{REFERENCES}

(1) Lee, N.; Kim, T.; Lim, J.-S.; Chang, I.; Cho, H. H. MetamaterialSelective Emitter for Maximizing Infrared Camouflage Performance with Energy Dissipation. ACS Appl. Mater. Interfaces 2019, 11, 21250-21257.

(2) Rodrigo, D.; Tittl, A.; John-Herpin, A.; Limaj, O.; Altug, H. SelfSimilar Multiresonant Nanoantenna Arrays for Sensing from Near- to Mid-Infrared. ACS Photonics 2018, 5, 4903-4911.

(3) Lochbaum, A.; Dorodnyy, A.; Koch, U.; Koepfli, S. M.; Volk, S.; Fedoryshyn, Y.; Wood, V.; Leuthold, J. Compact Mid-Infrared Gas Sensing Enabled by an All-Metamaterial Design. Nano Lett. 2020, 20, 4169-4176.

(4) Shi, N. N.; Tsai, C.-C.; Camino, F.; Bernard, G. D.; Yu, N.; Wehner, R. Keeping Cool: Enhanced Optical Reflection and Radiative Heat Dissipation in Saharan Silver Ants. Science 2015, 349, 298-301.

(5) Wang, T.; Li, P.; Chigrin, D. N.; Giles, A. J.; Bezares, F. J.; Glembocki, O. J.; Caldwell, J. D.; Taubner, T. Phonon-Polaritonic Bowtie Nanoantennas: Controlling Infrared Thermal Radiation at the Nanoscale. ACS Photonics 2017, 4, 1753-1760.

(6) Caldwell, J. D.; Glembocki, O. J.; Francescato, Y.; Sharac, N.; Giannini, V.; Bezares, F. J.; Long, J. P.; Owrutsky, J. C.; Vurgaftman, I.; Tischler, J. G.; Wheeler, V. D.; Bassim, N. D.; Shirey, L. M.; Kasica, R.; Maier, S. A. Low-Loss, Extreme Subdiffraction Photon Confinement via Silicon Carbide Localized Surface Phonon Polariton Resonators. Nano Lett. 2013, 13, 3690-3697.

(7) Muntasir Hossain, M.; Gu, M. Radiative Cooling: Principles, Progress, and Potentials. Adv. Sci. 2016, 3, 1500360.

(8) Kim, T.; Bae, J.-Y.; Lee, N.; Cho, H. H. Hierarchical Metamaterials for Multispectral Camouflage of Infrared and Microwaves. Adv. Funct. Mater. 2019, 29, 1807319.

(9) Koechlin, C.; Bouchon, P.; Pardo, F.; Jaeck, J.; Lafosse, X.; Pelouard, J.-L.; Haïdar, R. Total Routing and Absorption of Photons in Dual Color Plasmonic Antennas. Appl. Phys. Lett. 2011, 99, 241104.

(10) Granqvist, C. G.; Hjortsberg, A. Surfaces for Radiative Cooling: Silicon Monoxide Films on Aluminum. Appl. Phys. Lett. 1980, 36, 139-141.

(11) Zhai, Y.; Ma, Y.; David, S. N.; Zhao, D.; Lou, R.; Tan, G.; Yang, R.; Yin, X. Scalable-Manufactured Randomized Glass-Polymer Hybrid Metamaterial for Daytime Radiative Cooling. Science 2017, 355, $1062-1066$.
(12) Mandal, J.; Fu, Y.; Overvig, A. C.; Jia, M.; Sun, K.; Shi, N. N.; Zhou, H.; Xiao, X.; Yu, N.; Yang, Y. Hierarchically Porous Polymer Coatings for Highly Efficient Passive Daytime Radiative Cooling. Science 2018, 362, 315-319.

(13) Atiganyanun, S.; Plumley, J. B.; Han, S. J.; Hsu, K.; Cytrynbaum, J.; Peng, T. L.; Han, S. M.; Han, S. E. Effective Radiative Cooling by Paint-Format Microsphere-Based Photonic Random Media. ACS Photonics 2018, 5, 1181-1187.

(14) Fu, Y.; Yang, J.; Su, Y. S.; Du, W.; Ma, Y. G. Daytime Passive Radiative Cooler Using Porous Alumina. Sol. Energy Mater. Sol. Cells 2019, 191, 50-54.

(15) Li, T.; Zhai, Y.; He, S.; Gan, W.; Wei, Z.; Heidarinejad, M.; Dalgo, D.; Mi, R.; Zhao, X.; Song, J.; Dai, J.; Chen, C.; Aili, A.; Vellore, A.; Martini, A.; Yang, R.; Srebric, J.; Yin, X.; Hu, L. A Radiative Cooling Structural Material. Science 2019, 364, 760-763.

(16) Chen, G.; Wang, Y.; Qiu, J.; Cao, J.; Zou, Y.; Wang, S.; Jia, D.; Zhou, Y. Robust Inorganic Daytime Radiative Cooling Coating Based on a Phosphate Geopolymer. ACS Appl. Mater. Interfaces 2020, 12, 54963.

(17) Zhou, J.; Economon, E. N.; Koschny, T.; Soukoulis, C. M. Unifying Approach to Left-Handed Material Design. Opt. Lett. 2006, $31,3620-3622$.

(18) Lee, B. J.; Wang, L. P.; Zhang, Z. M. Coherent Thermal Emission by Excitation of Magnetic Polaritons Between Periodic Strips and a Metallic Film. Opt. Express 2008, 16, 11328-11336.

(19) Pradhan, J. K.; Gopal Achanta, V.; Agarwal, A. K.; Anantha Ramakrishna, S. Performance Enhancement due to a Top Dielectric Coating on a Metamaterial Perfect Absorber. Appl. Opt. 2020, 59, E118-E125.

(20) Cooper, C. T.; Rodriguez, M.; Blair, S.; Shumaker-Parry, J. S. Mid-Infrared Localized Plasmons Through Structural Control of Gold and Silver Nanocrescents. J. Phys. Chem. C 2015, 119, 11826-11832.

(21) Gillibert, R.; Malerba, M.; Spirito, D.; Giliberti, V.; Li, L.; Davies, A. G.; Linfield, E. H.; Baldassarre, L.; Colombelli, R.; Ortolani, M. Nanospectroscopy of a Single Patch Antenna Strongly Coupled to a Mid-Infrared Intersubband Transition in a Quantum Well. Appl. Phys. Lett. 2020, 117, 101104.

(22) Li, C.; Krachmalnicoff, V.; Bouchon, P.; Jaeck, J.; Bardou, N.; Haidar, R.; De Wilde, Y. Near-Field and Far-Field Thermal Emission of an Individual Patch Nanoantenna. Phys. Rev. Lett. 2018, 121, 243901.

(23) Chevalier, P.; Bouchon, P.; Jaeck, J.; Lauwick, D.; Bardou, N.; Kattnig, A.; Pardo, F.; Haïdar, R. Absorbing Metasurface Created by Diffractionless Disordered Arrays of Nanoantennas. Appl. Phys. Lett. 2015, 107, 251108.

(24) Chen, Y. N.; Todorov, Y.; Askenazi, B.; Vasanelli, A.; Biasiol, G.; Colombelli, R.; Sirtori, C. Antenna-Coupled Microcavities for Enhanced Infrared Photo-Detection. Appl. Phys. Lett. 2014, 104, 031113.

(25) Koechlin, C.; Bouchon, P.; Pardo, F.; Pelouard, J.-L.; Haïdar, R. Analytical Description of Subwavelength Plasmonic MIM Resonators and of their Combination. Opt. Express 2013, 21, 7025-7032.

(26) Fox, M. Optical Properties of Solids. Oxford Master Series in Physics; OUP Oxford, 2010.

(27) Joannopoulos, J.; Johnson, S.; Winn, J.; Meade, R. Photonic Crystals: Molding the Flow of Light, 2nd ed.; Princeton University Press, 2011.

(28) Rakić, A. D.; Djurisic, A. B.; Elazar, J. M.; Majewski, M. L. Optical Properties of Metallic Films for Vertical-Cavity Optoelectronic Devices. Appl. Opt. 1998, 37, 5271-5283.

(29) Querry, M. Optical Constants of Minerals and Other Materials from the Millimeter to the Ultraviolet; Report CRDEC-CR-88009; U.S. Army Armament, Munitions Chemical Command, 1987.

(30) Engheta, N. Circuits with Light at Nanoscales: Optical Nanocircuits Inspired by Metamaterials. Science 2007, 317, 16981702.

(31) Feng, R.; Qiu, J.; Liu, L.; Ding, W.; Chen, L. Parallel LC Circuit Model for Multi-Band Absorption and Preliminary Design of Radiative Cooling. Opt. Express 2014, 22, A1713-A1724. 
(32) Mason, J. A.; Smith, S.; Wasserman, D. Strong Absorption and Selective Thermal Emission from a Midinfrared Metamaterial. Appl. Phys. Lett. 2011, 98, 241105.

(33) Cui, Y.; Fung, K. H.; Xu, J.; Ma, H.; Jin, Y.; He, S.; Fang, N. X. Ultrabroadband Light Absorption by a Sawtooth Anisotropic Metamaterial Slab. Nano Lett. 2012, 12, 1443-1447.

(34) Addeo, A.; Monza, E.; Peraldo, M.; Bartoli, B.; Coluzzi, B.; Silvestrini, V.; Troise, G. Selective Covers for Natural Cooling Devices. IL Nuovo Cimento C 1978, 1, 419-429.

(35) Torgerson, E.; Hellhake, J. Polymer Solar Filter for Enabling Direct Daytime Radiative Cooling. Sol. Energy Mater. Sol. Cells 2020, 206, 110319.

(36) Nilsson, T. M. J.; Niklasson, G. A. Radiative Cooling During the Day: Simulations and Experiments on Pigmented Polyethylene Cover Foils. Sol. Energy Mater. Sol. Cells 1995, 37, 93-118.

(37) Pech-May, N. W.; Retsch, M. Tunable Daytime Passive Radiative Cooling Based on a Broadband Angle Selective Low-Pass Filter. Nanoscale Adv. 2020, 2, 249-255.

(38) Jackson, J. D. Classical Electrodynamics, 3rd ed.; Wiley: New York, NY, 1999.

(39) Horák, M.; Křápek, V.; Hrtoň, M.; Konečná, A.; Ligmajer, F.; Stöger-Pollach, M.; Šamořil, T.; Paták, A.; Édes, Z.; Metelka, O.; Babocký, J.; Sikola, T. Limits of Babinet's Principle for Solid and Hollow Plasmonic Antennas. Sci. Rep. 2019, 9, 4004.

(40) Xu, J.; Wang, A.; Dan, Y. Plasmonic Micropipe Spectral Filters in Mid-Infrared. Opt. Lett. 2019, 44, 4479-4482. 